\title{
Discrete Lyapunov function by means of a discrete residual energy function and stability analysis for discrete time physical systems
}

\author{
Cem Civelek ${ }^{1}$ \\ ${ }^{1}$ Türk-Alman Üniversitesi
}

August 1, 2021

\begin{abstract}
In this research paper beginning from the fundamental concepts, all the basic approach is introduced for short. Then after obtaining the discrete Lagrange-dissipative model ( $\{\mathrm{L}, \mathrm{D}\}$ - model for short) of a discrete time observed physical/engineering dynamic system, the model will be used to develope the discrete Hamiltonian together with discrete dissipation energy as discrete Lyapunov function and as such, a systematic method is proposed to obtain discrete Lyapunov function in form of a residual energy function for discrete (time) observed physical systems to analyze the discrete time system related to stability. Stability analysis of a discrete time observed physical dynamic system is performed using discrete Lyapunov function in form of a residual energy function consisting of Hamiltonian together with dissipation energy. Application of the method was shown using two discrete time physical examples, one of which is a coupled one and time dependent. This coupled physical discrete time example is analyzed related to stability using two different formulations, one form of which leads to a result.
\end{abstract}

\section{Hosted file}

Discrete Residual Energy Function.docx available at https://authorea.com/users/428557/ articles/532375-discrete-lyapunov-function-by-means-of-a-discrete-residual-energyfunction-and-stability-analysis-for-discrete-time-physical-systems 